\title{
A Comparison of Mine Seismic Discriminators Based on Features of Source Parameters to Waveform Characteristics
}

\author{
Ju Ma, Guoyan Zhao, Longjun Dong, Guanghui Chen, and Chuxuan Zhang \\ School of Resources and Safety Engineering, Central South University, Changsha 410083, China \\ Correspondence should be addressed to Longjun Dong; rydong001@csu.edu.cn
}

Received 2 October 2014; Accepted 27 December 2014

Academic Editor: Marcin A. Lutynski

Copyright (C) $2015 \mathrm{Ju} \mathrm{Ma}$ et al. This is an open access article distributed under the Creative Commons Attribution License, which permits unrestricted use, distribution, and reproduction in any medium, provided the original work is properly cited.

\begin{abstract}
To find efficient methods for classifying mine seismic events, two features extraction approaches were proposed. Features of source parameters including the seismic moment, the seismic energy, the energy ratio of S- to P-wave, the static stress drop, time of occurrence, and the number of triggers were selected, counted, and analyzed in approach I. Waveform characteristics consisting of two slope values and the coordinates of the first peak and the maximum peak were extracted as the discriminating parameters in approach II. The discriminating performance of the two approaches was compared and discussed by applying the Bayes discriminant analysis to the characteristic parameters extracted. Classification results show that $83.5 \%$ of the original grouped cases are correctly classified by approach I, and $97.1 \%$ of original grouped cases are correctly classified by approach II. The advantages and limitations pertaining to each classifier were discussed by plotting the event magnitude versus sample number. Comparative analysis shows that the proposed method of approach II not only has a low misjudgment rate but also displays relative constancy when the testing samples fluctuate with seismic magnitude and energy.
\end{abstract}

\section{Introduction}

Mining excavations induce elastic and then inelastic deformation within the surrounding rock mass. A seismic event is a sudden inelastic deformation within a given volume of rock. Having recorded and processed a number of seismic events within a given volume of interest $\Delta V$ over time $\Delta t$, one can then quantify the changes in the strain and stress regimes and in the rheological properties of the rock mass deformation associated with the seismic radiation $[1,2]$. However, a variety of dynamic processes in mines which radiate seismic waves are detected by the seismic monitoring systems and in general, seismograms generated by a development or production blast and a shear fracturing or a sudden slip on a surface of weakness are the majority of records [3-8]. As recorded quarry blasts may mislead scientific interpreting and lead to erroneous results in the analysis of seismic hazards in mines, standard processing of seismic monitoring data require these events to be separated. An automatic classifier is necessary to be developed to reduce the dramatically arduous task of finding to which class each recorded event belongs [9-17].
Many researches have been carried out on the topic of source location during the last decade. All techniques for source location methods can be classified under two conditions that require prior knowledge of the sonic speed of the structure [18] and methods that do not require such information [19-21]. However, few researches focused on the discrimination of mine seismic events in the past. Generally two steps, features extraction and statistical identification, could be separated from all those limited amount discriminators. Parameters characterizing the source (such as magnitude, potency, moment, energy, static stress drop, apparent stress, and apparent volume) and parameters directly extracted from the seismograms (including amplitude, polarization, frequency, correlation coefficient, and travel time) are the two categories of discriminating features. Statistical methodologies like fisher discriminant classifier, logistic regression, unascertained measurement, and neural networks have been occasionally carried out on mine seismic events identification and classification.

Malovichko selected the time of day, the repetition of waveforms, the high-frequency versus the low-frequency 


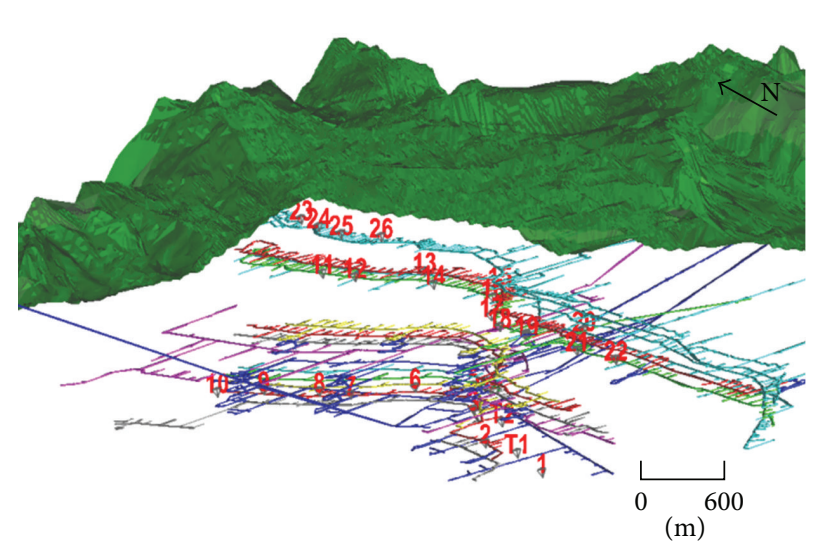

(a)

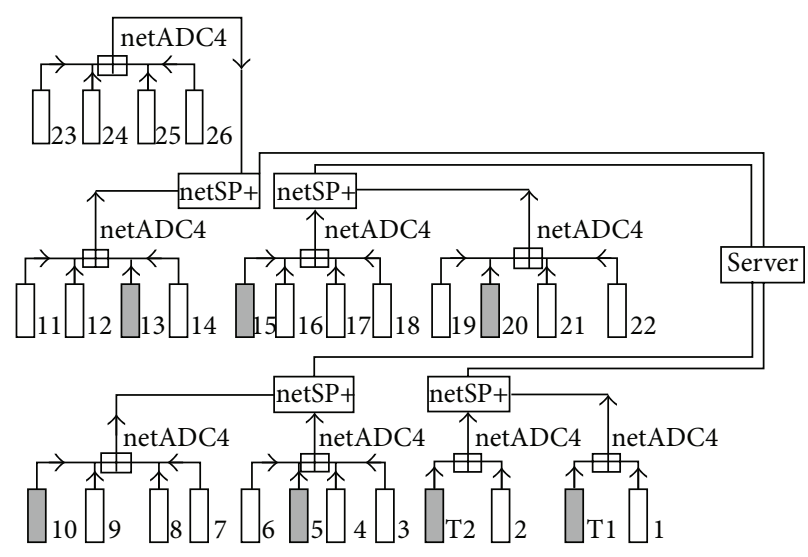

(b)

Figure 1: The microseismic monitoring system installed at Yongshaba mine: (a) isometric view of the ground surface, tunnels, and the microseismic monitoring system and (b) the configuration of the seismic network used in the project.

radiation and the radiation pattern as features and then established the Gaussian Maximum Likelihood Classification method for the classification [2]. This method provides a way to identify signals of different type, but the great amount of computation leads to low efficiency. Vallejos and McKinnon proposed the identification of seismic records in seismically active mines by considering the logistic regression and the neural network classification techniques. An efficient methodology was presented for applying these approaches to the classification of seismic records [3]. However, the calculation of seismic source parameters requires precise signal processing, namely expertise-required and time-consuming $\mathrm{P}$ - and S-wave hand-picking. Besides, the statistical classification models that only rely on source parameters show great difference in accuracy when applied to different mine sites.

Liu et al. proposed a synthesis method to identify microseismic event based on the triggering principle of short term average to long term average [9]. But very small fluctuations in the threshold would cause a great rate of misclassification since the threshold setting completely depends on experience. Zhu et al. decomposed the microseismic signals into 5 layers to gain specified frequency bands using wavelet analysis. Based on the box fractal dimensions and those specified frequency bands, 23-dimensional values of pattern recognition feature vector were established. The support vector machine, which was adopted to train, classify, and recognize, shows a correct identification rate of 94\% [10]. Jiang et al. presented a three-step strategy to achieve the classifying of local multichannels microseismic waveforms. The author extracted the time-frequency, the amplitude distribution, and the correlation coefficient as features and established an effective judgment mechanism [11]. However, the studies did not provide much attention to the huge workload of calculation in practical application. Their input data could not be obtained directly from the monitoring system and their calculation algorithm is very complicated. The efficiency of identification still needs further improvement since any algorithm must be as simple as possible in order to run on a small low-power microprocessor.
A classification method of mine blasts and microseismic events suing the starting-up features in seismograms was proposed. It is a method that presents less computation with a relatively low misjudgment rate. In this study, the Bayes discriminant classifier is applied to further analyse the "starting-up features." The differential discriminating performances acquired by means of extracting the features of, respectively source parameters and waveform, were compared and discussed.

\section{Data}

2.1. Engineering Background. The Yongshaba Mine is located at Gaobang, which is about $85 \mathrm{~km}$ northeast of Guiyang $\left(26^{\circ} 38^{\prime} \mathrm{N}, 106^{\circ} 37^{\prime} \mathrm{E}\right)$, Guizhou Province, PR China. It is the main operation base of the Guizhou Kailin Co. Ltd. with phosphate production capacity over 200,0000 tons/year. The current exploitation stopes are mainly scattered on levels of $1090 \mathrm{~m}$ to $840 \mathrm{~m}$ with a relative depth of $500 \mathrm{~m}$ to $700 \mathrm{~m}$ below the surface. Tens of millions of tons has cumulatively excavated employing open stoping mining method since initial operating in the 1950s.

The study region covers a volume of approximately $3000 \mathrm{~m} \times 300 \mathrm{~m} \times 350 \mathrm{~m}$, between the depths of $300 \mathrm{~m}$ and $700 \mathrm{~m}$ below the surface. The underground microseismic monitoring system, used to inform the evolution of the microfracture behavior, consists of 26 uniaxial and 2 triaxial geophones (Figure 1). The geophone manufactured by IMS holds a natural frequency of $14 \mathrm{~Hz}$ and a sampling rate of $6000 \mathrm{~Hz}$. Signals from various dynamic processes, fracturing in rock mass, production and development blasts, impacts, and vibration of machinery, all are being recorded by the microseismic monitoring system.

2.2. Databases. The sample databases used in this study consist of two parts: the subset of the signals of blast events and the subset of the signals of normal microseismic events. Each of the signals in the blast subset has been confirmed to be consistent with the blast operations according to 


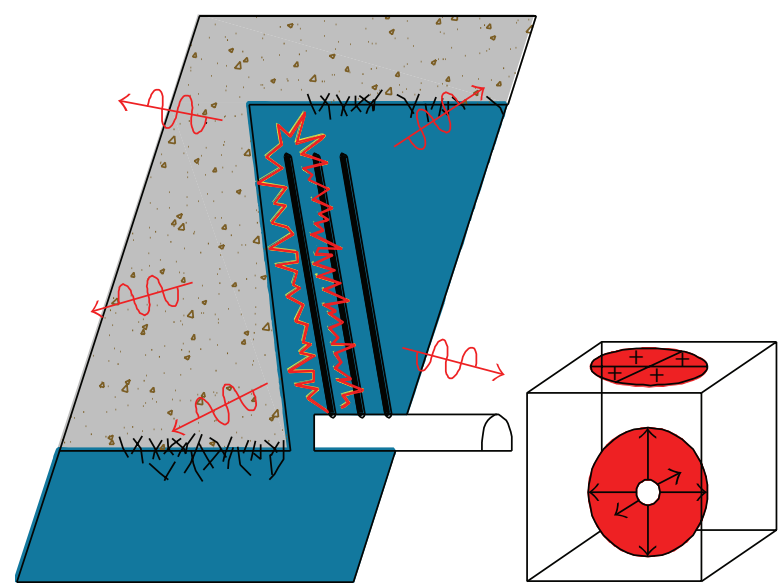

(a)

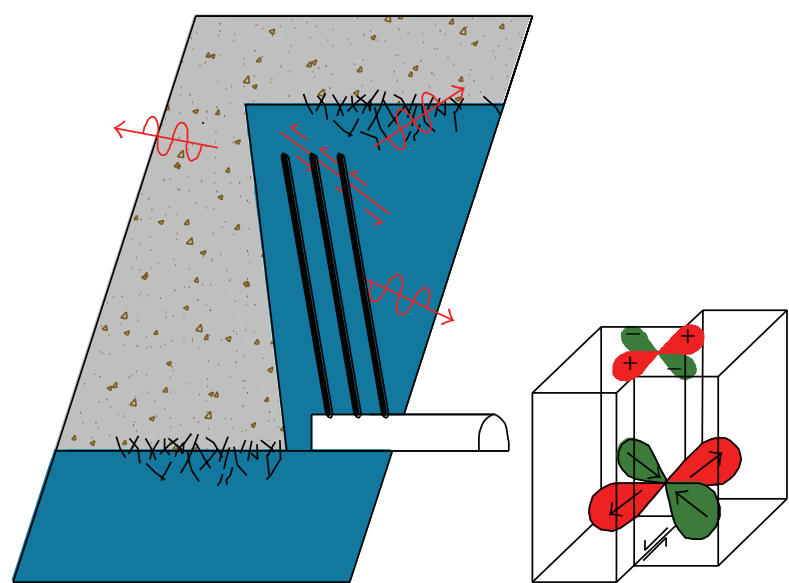

(b)

FIGURE 2: Two typical seismic source processes in mines, blast (a) and shear fracturing (b) (Malovichko 2012). Radiation pattern of an idealized explosion and of a strike-slip earthquake along a vertically dipping fault are shown correspondingly (Bormann, 2002). The arrows show the directions of compressional (outward, polarity +, red shaded) and dilatational (inward, polarity -, green shaded) motions in P-wave.

the time and location records provided by the mining workers. The subset of the microseismic events can be divided into two parts: those related to discrete, large-scale rock mass failures and those not resulting in observable rock mass damage. The observable ones also have been confirmed to be consistent with the fact. For the unobservable ones, they were processed manually by three independent processors. To aid in determining whether an event is a microseismic or not, the guide lines of an unequivocal classification were listed in Table 1. Only when the three discriminating results are exactly equivalent can the event be recruited into the database. Those events that could not be determined which type belongs to by manually approaches, were not enrolled into the sample database. The databases used in this paper contain a total of 103 seismic records with all seismic parameters calculated, from which 56 are labeled as normal events and the others are tagged as blasts.

\section{Discriminating Features}

3.1. Approach I: Source Parameters. A seismic event is the sudden release of potential or stored energy in the rock. The released energy is then radiated as seismic wave [7]. The two typical seismic source processes in mines (the blasts and the shear fracturing of the microseismic events) and its approximate radiation patterns are shown in Figure 2. It is obvious that the explosion radiates predominantly Pwaves outward directing compressional directions while the shear fracturing or slip on a surface radiates $S$-waves that are stronger than P-waves. These characteristics can be used to identify the type of source process and to discriminate between blasts and microseismic events [2].

3.1.1. Seismic Moment. The basic characteristics of radiation can be described by a seismic moment tensor. This tensor represents a set of fictitious dipoles acting on a point in the source area. The moment tensor makes it possible to describe the low-frequency amplitudes and polarities of seismic waves. Inverting the moment tensor from the observed waveforms and analysis of its components allows the discrimination of blasts from slip-type and, even, from crush-type events $[2,13,14]$. The logarithm of the seismic moment is considered as the feature of radiation.

Figure 3(a) shows that the values of the $\log (M)$ at the points with the highest probability density are 10.0 for blasts and 8.0 for microseismic events. The seismic moment is a relative useful performing discriminating feature as it provides a relatively large separation between normal events and blasts.

3.1.2. Seismic Energy. The energy release during rock fracturing and frictional sliding comes from the transformation of elastic strain into inelastic strain [14]. This transformation may occur at different rates ranging from slow creep-like events to very fast dynamic seismic events. The average velocity of deformation at the source is up to a few meters per second. Unlike the dynamic sources of the same size, the slow type events have long time duration at the source and thus radiate predominantly lower frequency waves. In terms of fracture mechanics, the slower the rupture velocity is, the less energy the event radiates. A quasi-static rupture would radiate practically no energy. Similarly for blasting, smaller blasts make smaller changes to the rock mass or more gradual stress changes, so that the response is less dynamic [12-16].

Observations from the probability density distribution (Figure 3(b)) indicate that the seismic energy is one of the best performing discriminating features. The value of $\log (E)$ distributed from -2 to 2 for microseismic events and 0 to 6 for blasts. More than $65 \%$ of the blasts events could be accurately identified in terms of this single indicator. 
TABLE 1: Guidelines for mine seismic events classification manually.

\begin{tabular}{ll}
\hline Characteristics & Description \\
\hline $\begin{array}{l}\text { The presence of delays of the blast } \\
\text { Blasting seismogram } \\
\text { with upper envelope }\end{array}$ & $\begin{array}{l}\text { Blasts, especially stope firings, have multiple delays, which are } \\
\text { expressed in the seismogram as similar signals repeating closely } \\
\text { within a short time interval. The practice of decides whether an event } \\
\text { is a blast or a microseismic event is based on the repetition feature. } \\
\text { Besides, seismograms capturing a blast will have a monotonically } \\
\text { decreasing tail commonly. For the waveform of a microseismic signal, } \\
\text { a large amplitude difference exists between the maximum peak and } \\
\text { the peak closest behind, without a gradual decrease. }\end{array}$ \\
\end{tabular}

The steeper rise in the energy curve of the normal event

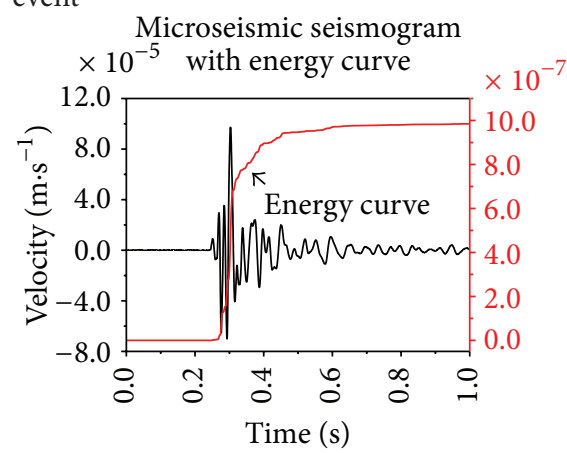

Seismograms capturing a microseismic event usually associated with shear fracturing and have an S-wave arrival more obvious than in the cases of blasts due to the source of the latter are usually in the focal mechanism of expansion and compression. Furthermore, blasting events will typically have a consistent gradual rise in their energy curve while seismic events will tend to have a steeper, more distinct, rise in the energy curve, and the energy curves for blasting events can be loosely compared to a positive sloping flat line.

The time of occurrence

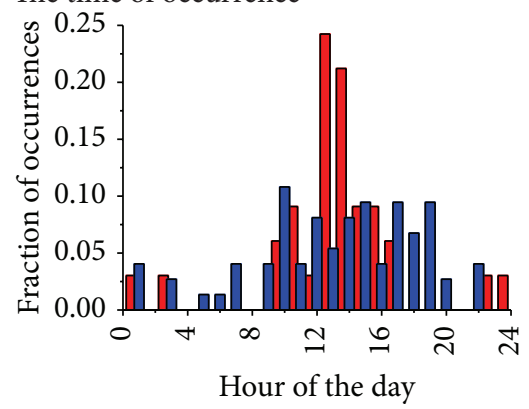

Another way to eliminate blasts from the microseismic catalogue is to apply the time filters (i.e., generally mines have prescribed blasting times, and the events that does not occur at the blasting time are marked as microseismic events). Two main daily blasting shifts are observed from the typical diurnal chart of Chinese mines, hours between 10 16 (stope firings) and 23 1 (development firings), each of which triggers an increase in seismicity. It has to be classified referring to other features for the cases that recorded during the blasting time and located close to the blasting area.

- Blasts

- Microseismic events

The dominant frequency

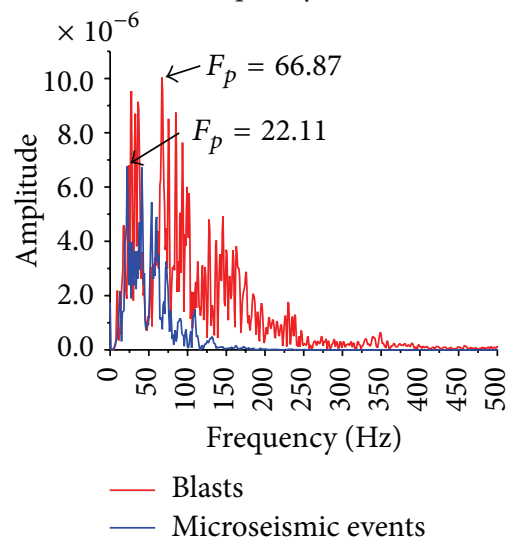

A large number of actual observations and analysis show that blasts or explosions usually radiate higher frequency waves compared to normal microseismic events. The statistics show that the value of the dominant frequency of the microseismic events varies from 10 to $100 \mathrm{~Hz}$ and from 70 to $260 \mathrm{~Hz}$ for blasts. In addition, blasting events will typically not be well matched to the Brune's model curve as plotted in the Stacked Spectra Plot within TRACE (the software provided by IMS). Spectral analysis is often considered as a relative effective method to distinguish blasts from microseismic events.

- Microseismic events 
TABle 1: Continued.

\begin{tabular}{ll}
\hline Characteristics & Description \\
\hline The maximum amplitude & $\begin{array}{l}\text { The maximum amplitude of the waveform, which characterizes the } \\
\text { size of energy released, is also considered as an important reference } \\
\text { for the recognition of blasts and microseismic events. Generally the } \\
\text { maximum vibration velocity of microseismic events is about } \\
10^{-5} \mathrm{~m} \cdot \mathrm{s}^{-1} \text { far less than } 10^{-3} \mathrm{~m} \cdot \mathrm{s}^{-1} \text { of the blasting. However, this } \\
\text { approach cannot be considered as universally applicable since it is } \\
\text { possible to have a scenario where a blast triggers shear fracturing that } \\
\text { radiates stronger seismic signals than the blast itself. }\end{array}$ \\
\\
\hline
\end{tabular}

3.1.3. S: P Energy Ratio. There are several seismic phases, but only $\mathrm{S}$-waves and $\mathrm{P}$-waves are commonly recorded in mining induced seismicity. P-waves, or compressive waves, are the first ones which arrive at receivers. When seismic waves propagate, they carry energy from the source of the shaking outward in all directions. The ratio between S-wave energy and P-wave energy can be an indicator of seismic source mechanism [4]. Seismic energy is proportional to the integral of the square of the vectorial sum of the velocity waveform and can be calculated separately for the P-wave and S-wave [7]. For fault-slip type events in seismology (earthquakes), there is considerably more energy in the S-wave than in the Pwave with the ratio of the S-wave energy to the P-wave energy frequently greater than 10 [12]. Urbancic et al. noted that for nonshear seismic source mechanisms, there would be a deficiency in S-wave energy or relatively more P-wave energy than for shearing events. For nonshearing event mechanisms, such as strain-bursting, tensile failure, and volumetric rock mass fracturing, the ratio of S-wave energy to P-wave energy is frequently in the range of 3 or less [14].

Observations from Figure 3(c) point out that the most probability of the $\log \left(E_{\mathrm{S}} / E_{\mathrm{P}}\right)$ falls in the interval of 0.0 to 2.0 for microseismic events and -1.0 to 4.0 for blasts. It was obvious that the $\mathrm{S}: \mathrm{P}$ energy ratio performs not well in this mine site since too small separation was provided.

3.1.4. Static Stress Drop. Shearer defined the term-stress drop, $\Delta \sigma$, as the average difference between the stress across the fault before and after an earthquake [17]. There are several different methods of determining the stress drop, of which some use records of ground velocity and ground acceleration. Stress drops can vary considerably from event to event. For microseismic events in Yongshaba mine, the range of $\log (\mathrm{SSD})$ is from 2.5 to 4.5 and 3.0 to 6.5 for blasts (Figure $3(\mathrm{~d})$ ). This parameter plays a tremendous role in Yongshaba mines seismic discrimination.
3.1.5. Time of Occurrence. Mining-induced seismic events occur most of the time in the ultimate proximity of mine workings and concentrate during blasting time. Statistical results show that almost all the blasts occur at 10:00 to 15:00 and rarely occur in other time (Figure 3(e)). However, a large number of microseismic events take place at this time simultaneously. The performance of the "time of occurrence" is discounted.

3.1.6. Number of Triggers. The Number of triggers is affected by many factors. Mine layout, geological features, seismic locations, and the sensor array all are likely to affect the number of triggered sensors. It should be guaranteed that the potential areas of microseismic occurring and blasting operations are enclosed inside the sensor array. Only in this way can the "number of triggers" reflect the pattern of seismic wave propagation and the scale of seismic energy. Figure 3(f) shows blasting events usually triggering more sensors than normal microseismic events. The discriminating feature of the "number of triggers" provides a relatively medium separation between normal events and blasts.

3.2. Approach II: Waveform Features. The classification method of mine blasts and microseismic events suing the starting-up features in seismograms was proposed. Signals from databases of different event types were drawn into a unified coordinate system. All waveform sections are starting at the point of each P-wave first arrival and ending in their first peak points (Figure 4(a)). It is noticed that the startingup angle of the two types tends to be concentrated into two different intervals. Since it is difficult to calculate the starting-up angle directly due to the inaccuracy of P-wave arrival's picking, the slope value of the starting-up trend line obtained from linear regression was proposed to substitute the angle. Two slope values associated with the coordinates 


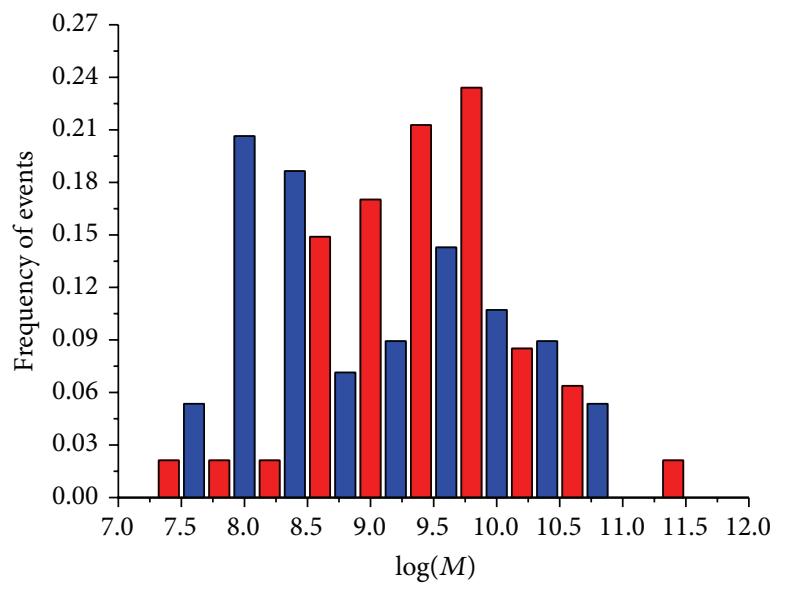

(a)

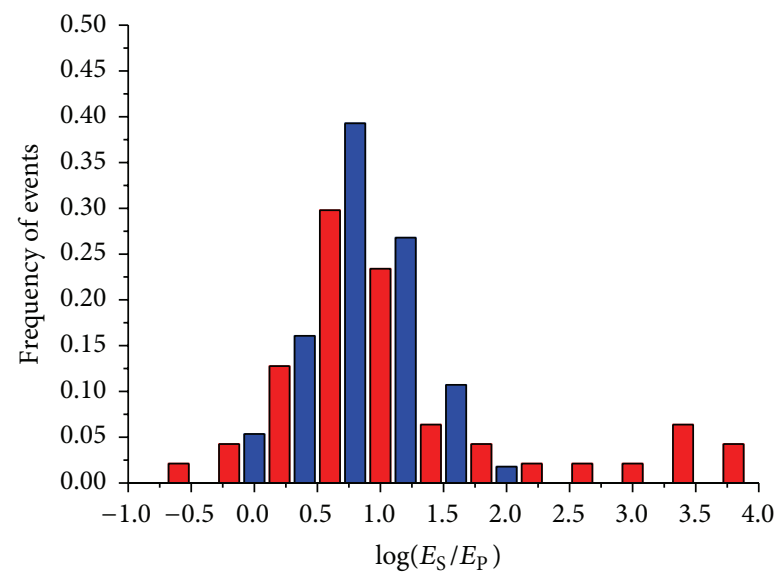

(c)

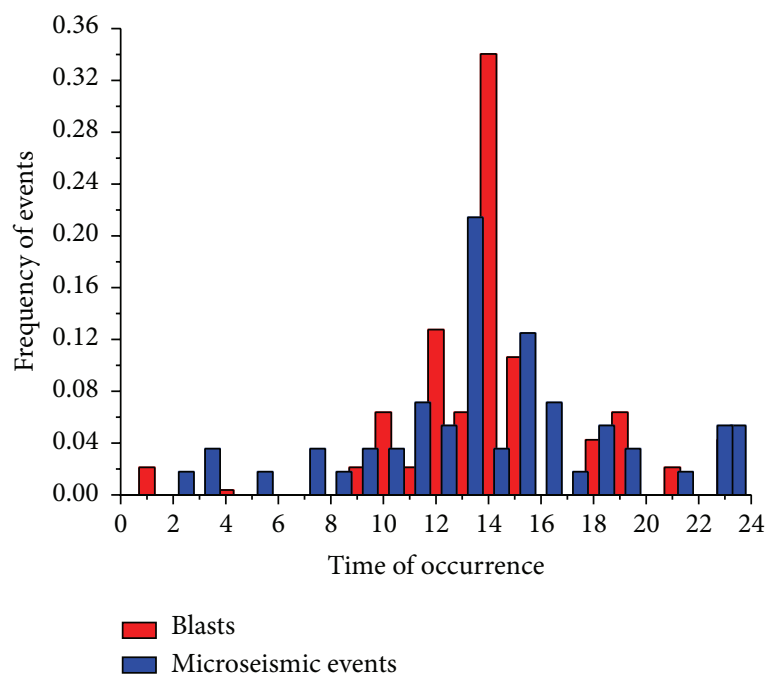

(e)

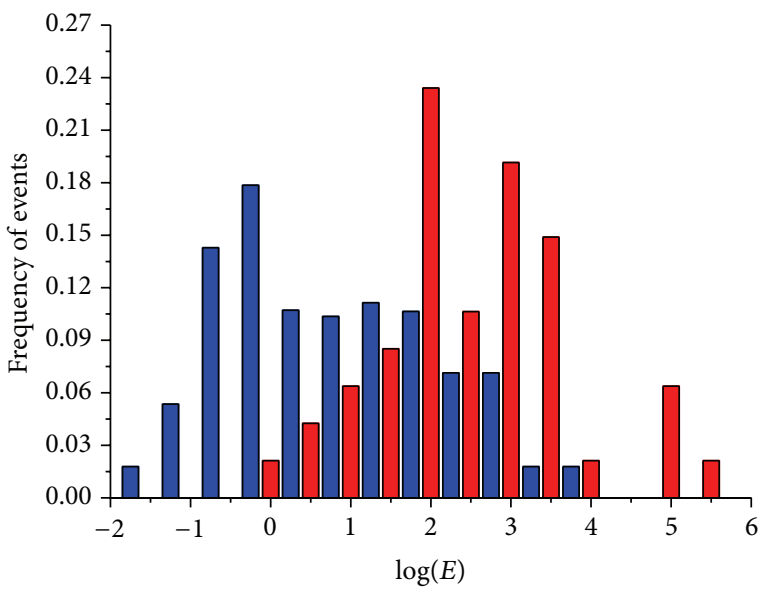

(b)

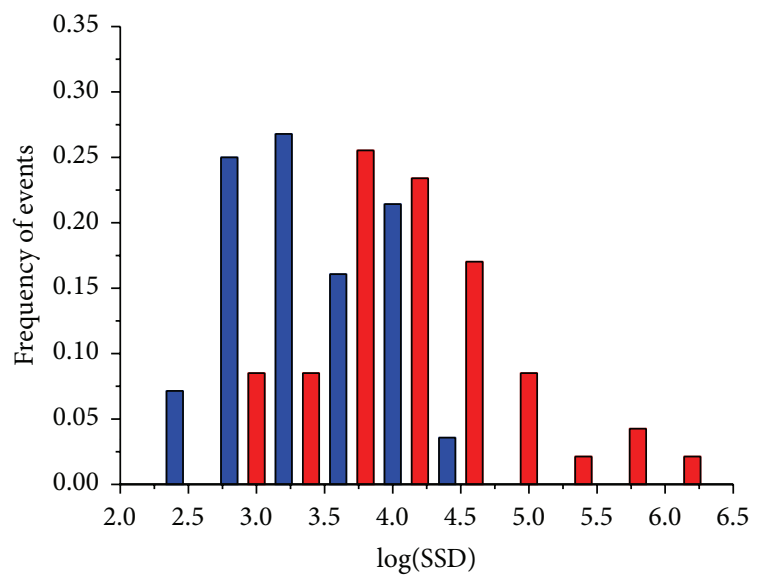

(d)

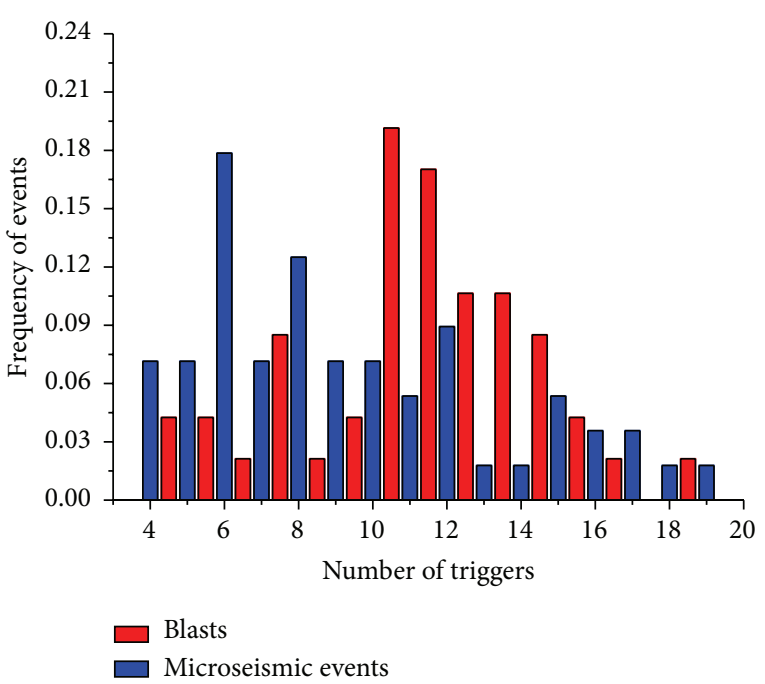

(f)

FIGURE 3: Distributions of the measured features for the database are displayed as histograms. The seismic energy is the best performing discriminating feature as it provides maximum separation between normal events and blasts. The feature "time of occurrence" provides the slightest separation between blasts and normal events. 


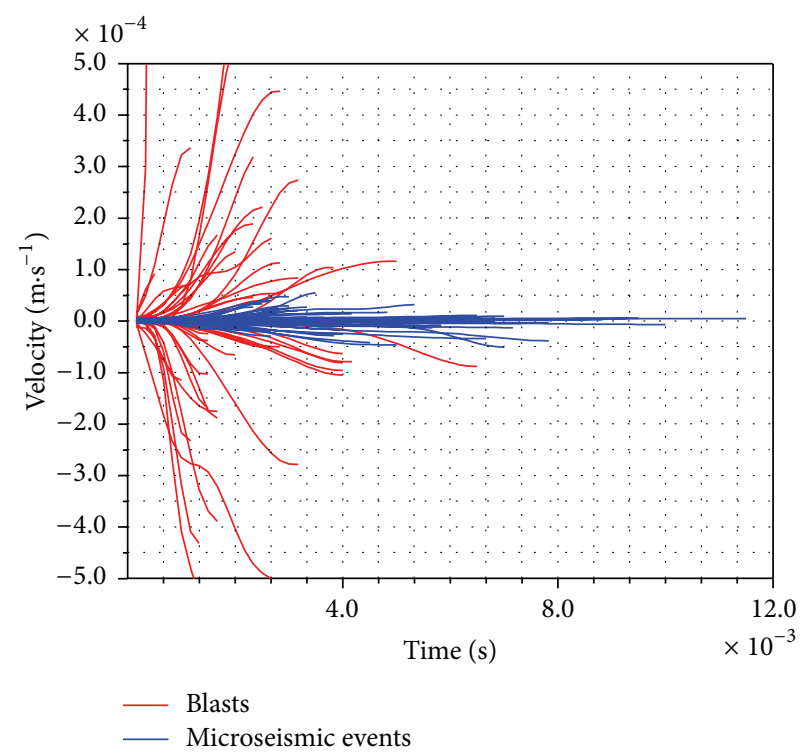

(a)

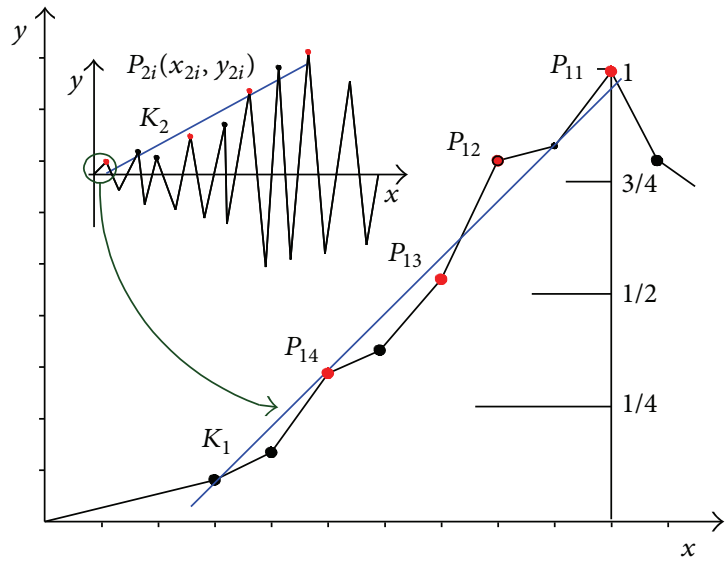

(b)

FIGURE 4: Comparison chart of signal starting-up before first peak within blasts and microseismic events (frame (a)) and the schematic diagram of data points selecting and the trend line constructed by linear regression (frame (b), solid circles represent sampling points, and the reds represent the selected). The $x$ - and $y$-axis in frame (b) represent the time and the amplitude axis in frame (a).

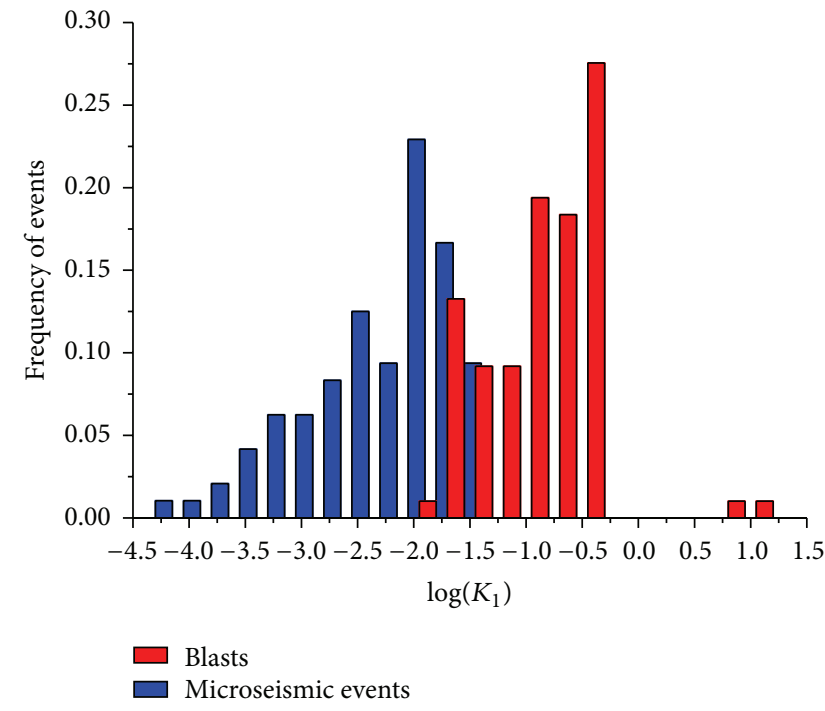

(a)

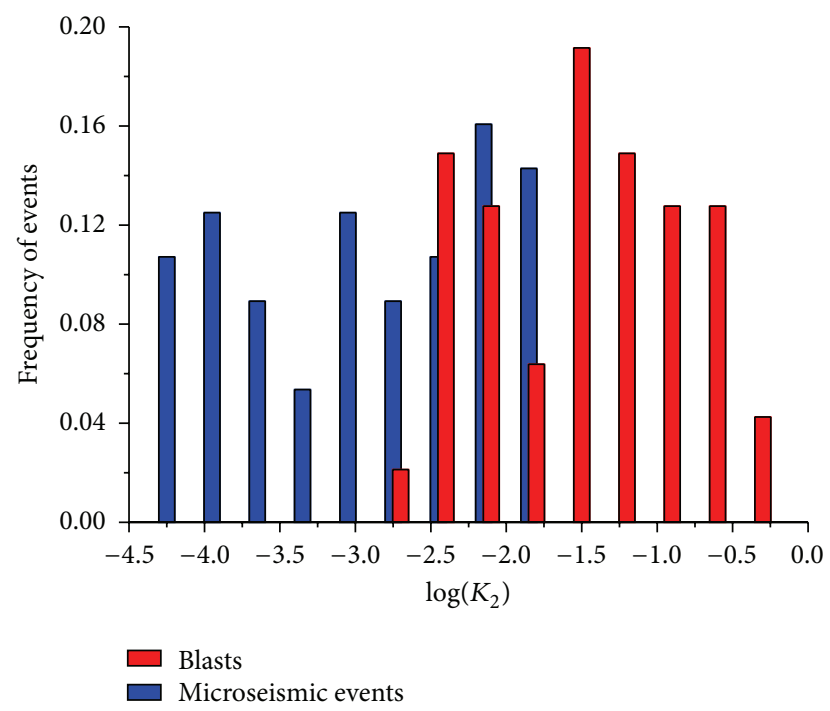

(b)

FIGURE 5: Comparison chart of slope value of the two starting-up trend lines within waveforms of blasts and microseismic events. Histograms are used to illustrate the behavior of the discriminating features.

of the first peak and the maximum peak were extracted as the characteristic parameters.

Set $K_{1}$ as the absolute value of the slope of the trend line of the waveform section that is from the P-wave arrival to the first peak, and set $K_{2}$ as the absolute value of the slope of the trend line of the waveform section that is from the Pwave arrival to the maximum peak. The two trend lines are constructed by linear regression based on four points. The method of the features extraction and their performance are displayed in Figures 4 and 5.

\section{Method}

Bayes discriminant is a branch of modern statistics with the basic hypothesis that some certain cognitions of the studying collectivity had been received before extracting samples. Generally using the priori probability to describe the level of awareness, and then the posterior probability was obtained by modifying it [22-26].

Suppose $\mathbf{G}=\left(\mathbf{X}_{1}, \mathbf{X}_{2}, \ldots, \mathbf{X}_{\mathbf{p}}\right)^{T}$ is a collectivity with $p$ member indexes, and $f_{1}(\mathbf{X})$ and $f_{2}(\mathbf{X})$ are the distribution 
density functions of the two collectivities: $\mathbf{G}_{\mathbf{1}}$ which refers to blasts and $\mathbf{G}_{2}$ which refers to microseismic events. Indexes $\mathbf{X}_{1}, \mathbf{X}_{2}, \mathbf{X}_{3}, \mathbf{X}_{4}$, and $\mathbf{X}_{5}$ represent the characteristic parameters, respectively.

The priori probability of $\mathbf{G}_{\mathbf{1}}$ and $\mathbf{G}_{\mathbf{2}}$ are calculated by the following formulas:

$$
p_{1}=P\left(\mathbf{G}_{1}\right)=\frac{n_{1}}{n_{1}+n_{2}}, \quad p_{2}=P\left(\mathbf{G}_{2}\right)=\frac{n_{2}}{n_{1}+n_{2}},
$$

where $n_{1}$ and $n_{2}$ are the number of training samples belonged to the collectivity $\mathbf{G}_{\mathbf{1}}$ and $\mathbf{G}_{\mathbf{2}}$.

Set $\Sigma_{1}, \Sigma_{2}$, and $\Sigma$ as the covariance matrix of $\mathbf{G}_{1}, \mathbf{G}_{2}$, and $\mathbf{G}$. The Bayes discriminant function can be expressed as follows when $\Sigma_{1}=\Sigma_{2}=\Sigma$ :

$$
W_{j}(\mathbf{X})=\left(\boldsymbol{\Sigma}^{-\mathbf{1}} \boldsymbol{\mu}_{\mathbf{j}}\right)^{T}-0.5 \boldsymbol{\mu}_{\mathbf{j}}^{T} \boldsymbol{\Sigma}^{-\mathbf{1}} \boldsymbol{\mu}_{\mathbf{j}}+\ln p_{j}, \quad j=1,2,
$$

where $\boldsymbol{\mu}_{\mathbf{j}}$ is the mean vectors of $\mathbf{G}_{\mathbf{j}}$ and the generalized squared distance function can be obtained as

$$
d_{j}^{2}(\mathbf{X})=\left(\mathbf{X}-\boldsymbol{\mu}_{\mathbf{j}}\right)^{T} \boldsymbol{\Sigma}^{-\mathbf{1}}\left(\mathbf{X}-\boldsymbol{\mu}_{\mathbf{j}}\right)-2 \ln p_{j} .
$$

The a posteriori probability function can be obtained as follows:

$$
P\left(\mathbf{G}_{\mathbf{j}} \mid \mathbf{X}\right)=\frac{p_{j} f_{j}(\mathbf{X})}{p_{1} f_{1}(\mathbf{X})+p_{2} f_{2}(\mathbf{X})} .
$$

Since $\mathbf{p}_{\mathbf{j}} f_{j}(\mathbf{X}) \propto \exp \left[-0.5 d_{j}^{2}(\mathbf{X})\right]$, then

$$
P\left(\mathbf{G}_{\mathbf{j}} \mid \mathbf{X}\right)=\frac{\exp \left[-0.5 d_{j}^{2}(\mathbf{X})\right]}{\exp \left[-0.5 d_{1}^{2}(\mathbf{X})\right]+\exp \left[-0.5 d_{1}^{2}(\mathbf{X})\right]} .
$$

Normally $\boldsymbol{\mu}_{1}, \boldsymbol{\mu}_{2}$, and $\boldsymbol{\Sigma}$ are unknown and their estimation values $\widehat{\boldsymbol{\mu}}_{1}, \widehat{\boldsymbol{\mu}}_{2}$, and $\widehat{\boldsymbol{\Sigma}}$ can be obtained from the training samples; then

$$
\widehat{d}_{j}^{2}(\mathbf{X})=\left(\mathbf{X}-\widehat{\boldsymbol{\mu}}_{\mathbf{j}}\right)^{T} \widehat{\boldsymbol{\Sigma}}^{-1}\left(\mathbf{X}-\widehat{\boldsymbol{\mu}}_{\mathbf{j}}\right)-2 \ln p_{j} .
$$

The estimation of a posteriori probability function is

$$
\widehat{P}\left(\mathbf{G}_{\mathbf{j}} \mid \mathbf{X}\right)=\frac{\exp \left[-0.5 \widehat{d}_{j}^{2}(\mathbf{X})\right]}{\exp \left[-0.5 \widehat{d}_{1}^{2}(\mathbf{X})\right]+\exp \left[-0.5 \widehat{d}_{1}^{2}(\mathbf{X})\right]} .
$$

Bayes discriminant criterion can be expressed as

$$
\begin{aligned}
& \mathbf{X} \in \mathbf{G}_{\mathbf{1}}, \text { when } \widehat{P}\left(\mathbf{G}_{\mathbf{1}} \mid \mathbf{X}\right) \geq \widehat{P}\left(\mathbf{G}_{\mathbf{2}} \mid \mathbf{X}\right), \\
& \mathbf{X} \in \mathbf{G}_{\mathbf{2}}, \quad \text { when } \widehat{P}\left(\mathbf{G}_{\mathbf{1}} \mid \mathbf{X}\right)<\widehat{P}\left(\mathbf{G}_{\mathbf{2}} \mid \mathbf{X}\right) .
\end{aligned}
$$

To estimate the reliability of the discriminator, the resubstitution method was used to calculate the misdiscrimination rate. All the training samples were regarded as the new samples and resubstituted into the classifier. The rate of misjudgment can be evaluated by the following index $\widehat{\alpha}$ :

$$
\widehat{\alpha}=\frac{n_{12}+n_{21}}{n_{1}+n_{2}},
$$

where $n_{12}$ is the number of training samples regarded to be $\mathbf{G}_{2}$ which belong to collectivity of $\mathbf{G}_{1}$ actually and $n_{21}$ is the number of training samples discriminated as $\mathbf{G}_{\mathbf{1}}$ but belongs to $\mathbf{G}_{\mathbf{2}}$ in fact.
TABLE 2: Prior probabilities for groups.

\begin{tabular}{lccc}
\hline \multirow{2}{*}{ Type } & Prior & \multicolumn{2}{c}{ Cases used in analysis } \\
& & Unweight & Weighted \\
\hline Blast & 0.5 & 47 & 47.0 \\
Microseismic event & 0.5 & 56 & 56.0 \\
\hline Total & 1.0 & 103 & 103.0 \\
\hline
\end{tabular}

\section{Results}

The aim of the present study is to compare the two approaches for event accurate identification of different classes. The Bayes discriminant models for signal identification are established after developing the theory discussed above to the 103 sets of samples selected. The prior probabilities for different groups and the classification function coefficients are listed in Tables 2 and 3 .

As can be seen from the classification functions coefficients, the features of source parameters are in the following order according to their importance in the accurate identification of microseismic events: the seismic moment, the static stress drop, the seismic energy, the S:P energy ratio, the number of triggers, and the time of occurrence. According to their importance in the accurate identification of microseismic events, the waveform characteristics are in order of the time of the first peak arrival $\left(x_{11}\right)$, the amplitude of the maximum peak $\left(y_{21}\right)$, the slope value of the first trend line $\left(K_{1}\right)$, the slope value of the second trend line $\left(K_{2}\right)$, the amplitude of the first peak $\left(y_{11}\right)$, and the time of the maximum peak arrival $\left(x_{21}\right)$.

The classification results show that $83.5 \%$ of original grouped cases are correctly classified by approach I, and 97.1\% of original grouped cases are correctly classified by approach II (Table 4). The results show that the second features extraction approach (waveform characteristics) has higher accuracy. Although the input data used for classification in approach II cannot be given directly by the monitoring systems, the calculation process of those characteristic parameters are not complicated.

These misclassified events are labeled in Figure 6 according to the order of moment magnitude. Figure 6 shows the misclassified cases of both approaches falling without specific magnitude intervals. It is concluded that the error rate is not affected by the seismic magnitude or the scale of seismic energy. Although approach I has a higher misclassified rate with a more complex computation, it has been widely used in many mines. Approach II is a new straight-forward method with lower error rate and got very good application in the Yongshaba mine. But in other mines or in more complex cases (mines existing variety of dynamic processes which radiate seismic waves, such as shock and vibrations induced by orepass), the feasibility of this approach may need further improvements.

\section{Conclusion}

(1) The considerations and the criteria of manual identification of blasts and microseismic events were summarized 
TABLE 3: Classification function coefficients.

\begin{tabular}{lccccccc}
\hline Approach I & $\log (M)$ & $\log (E)$ & $\log \left(E_{\mathrm{S}} / E_{\mathrm{P}}\right)$ & $\log (\mathrm{SSD})$ & TOO $^{\mathrm{a}}$ & NOT $^{\mathrm{b}}$ & Constant \\
\hline Blast & 18.229 & -9.827 & 2.964 & 18.790 & 0.625 & -1.495 & -11.242 \\
Microseismic & 18.248 & -9.761 & 2.410 & 16.133 & 0.557 & -1.596 & -100.464 \\
\hline Approach II & $\log \left(x_{11}\right)$ & $\log \left(y_{11}\right)$ & $\log \left(K_{1}\right)$ & $\log \left(x_{21}\right)$ & $\log \left(y_{21}\right)$ & $\log \left(K_{2}\right)$ & $\operatorname{Constant}$ \\
\hline Blast & -92.588 & 3.878 & -8.471 & -3.704 & -33.644 & -4.304 & -186.187 \\
Microseismic & -91.487 & 2.263 & -10.915 & -0.344 & -32.830 & -7.053 & -193.783 \\
\hline
\end{tabular}

${ }^{\mathrm{a}}$ TOO presents the time of occurrence; ${ }^{\mathrm{b}}$ NOT presents the number of triggers.

TABLE 4: Classification results.

\begin{tabular}{|c|c|c|c|c|c|}
\hline \multirow{2}{*}{ Type } & \multirow{2}{*}{\multicolumn{2}{|c|}{$\begin{array}{l}\text { Predicted group membership } \\
\text { (approach I) }\end{array}$}} & \multirow{2}{*}{\multicolumn{2}{|c|}{$\begin{array}{l}\text { Predicted group membership } \\
\text { (approach II) }\end{array}$}} & \multirow{2}{*}{ Total } \\
\hline & & & & & \\
\hline \multicolumn{6}{|l|}{ Count } \\
\hline Blasts & 39 & 8 & 46 & 1 & 47 \\
\hline Microseismic events & 9 & 47 & 2 & 54 & 56 \\
\hline \multicolumn{6}{|l|}{$\%$} \\
\hline Blasts & 83.0 & 17.0 & 97.9 & 2.4 & 100.0 \\
\hline Microseismic events & 16.1 & 83.9 & 3.6 & 96.4 & 100.0 \\
\hline
\end{tabular}

$83.5 \%$ of original grouped cases are correctly classified by approach I, and $97.1 \%$ of original grouped cases are correctly classified by approach II.

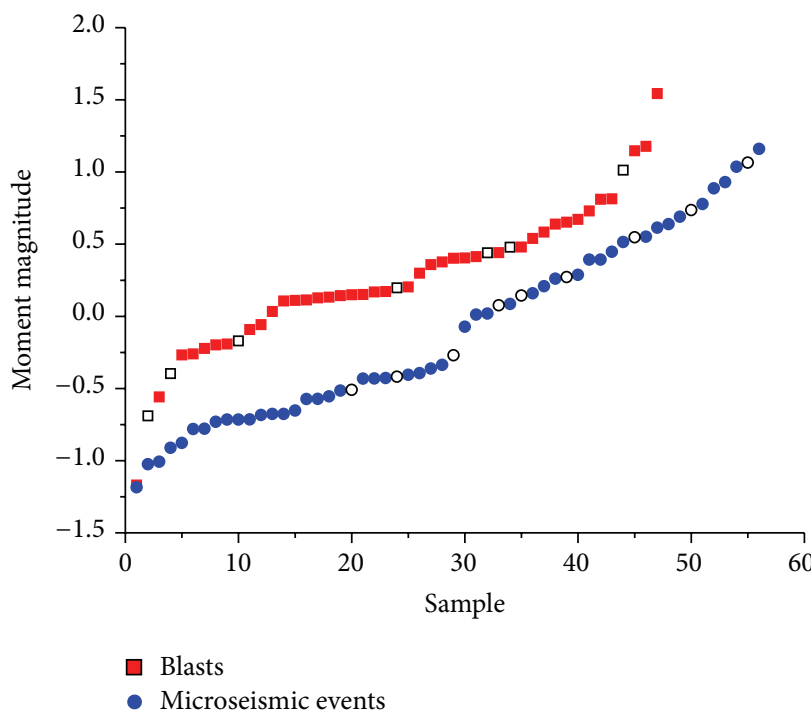

(a)

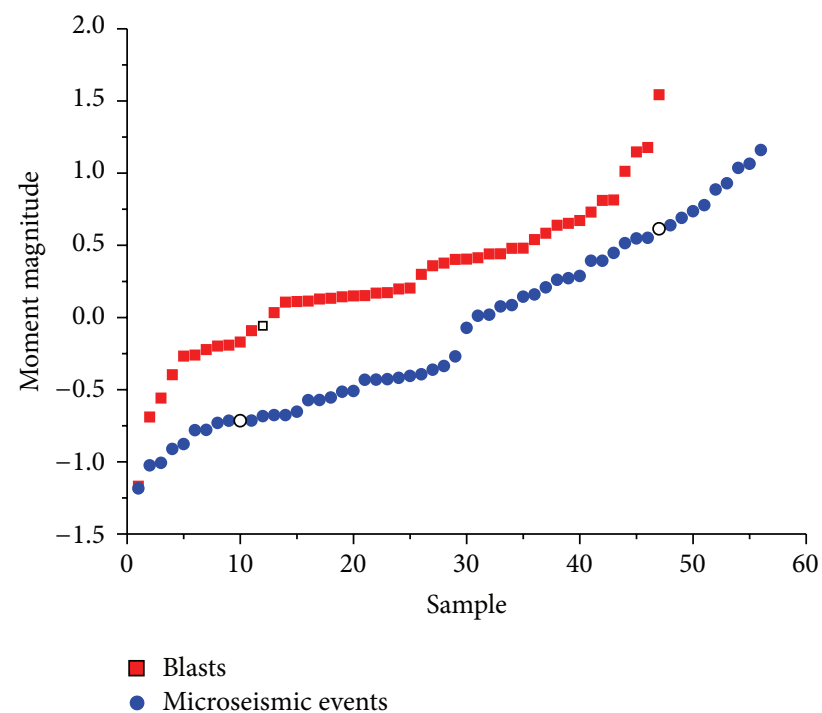

(b)

FiguRE 6: Events that are misclassified: frame (a) misclassified by approach I and frame (b) misclassified by approach II. The hollow ones represent the misclassified cases.

based on experiences. The guide lines listed in this study cover well the majority of all the encountered situations in seismic data processing. The sample databases of the two types' events were established by three independent processors with reference to these guidelines.

(2) Two features extraction approaches were proposed. Features of source parameters including the seismic moment, the seismic energy, the energy ratio of S- to P-wave, the static stress drop, the time of occurrence, and the number of triggers were selected, counted, and analyzed in approach I.
Waveform characteristics consisting of two slope values and the coordinates of the first peak and the maximum peak were extracted as the discriminating parameters in approach II.

(3) The discriminating performance of the two approaches were compared and discussed by applying the Bayes discriminant analysis to the characteristic parameters extracted. The classification results show that $83.5 \%$ of the original grouped cases were correctly classified by approach I, and $97.1 \%$ of original grouped cases were correctly classified by approach II. 
(4) Comparative analysis shows that the misclassified cases of the two approaches all fall without specific magnitude intervals. The error rate is not affected by the seismic magnitude. Although approach II has got very good application in the Yongshaba mine, the feasibility of this approach may need further improvements in other mines or in more complex cases, mines existing in variety of dynamic processes which radiate seismic waves, such as shock and vibrations induced by orepass.

\section{Conflict of Interests}

The authors declare that there is no conflict of interests regarding the publication of this paper.

\section{Acknowledgment}

The authors gratefully acknowledge the financial support of National Natural Science Foundation of China (no. 51374244, 11447242) and the Fundamental Research Funds of Central South University (no. 2013zzts058).

\section{References}

[1] A. J. Jager and J. A. Ryder, A Handbook on Rock Engineering Practice for Tabular Hard Rock Mines, Creda Communications, Cape Town, South Africa, 1999.

[2] D. Malovichko, "Discrimination of blasts in mine seismology," in Proceeding of the Deep Mining, Australian Centre for Geomechanics, Perth, Australia, 2012.

[3] J. A. Vallejos and S. D. McKinnon, "Logistic regression and neural network classification of seismic records," International Journal of Rock Mechanics and Mining Sciences, vol. 62, pp. 8695, 2013.

[4] S. J. Gibowicz and A. Kijko, An Introduction to Mining Seismology, Academic Press, San Diego, Calif, USA, 1994.

[5] K. Larsson, Seismicity in Mines: A Review, Luleå University of Technology, 2004.

[6] P. Bormann, New Manual of Seismological Observatory Practice, GFZ, Potsdam, Germany, 2002.

[7] A. J. Mendecki, Seismic Monitoring in Mines, Chapman \& Hall, London, UK, 1997.

[8] L.-J. Dong, X.-B. Li, Z.-L. Zhou, G.-H. Chen, and J. Ma, “Threedimensional analytical solution of acoustic emission source location for cuboid monitoring network without pre-measured wave velocity," Transactions of Nonferrous Metals Society of China, vol. 25, no. 1, pp. 293-302, 2015.

[9] C. Liu, C.-A. Tang, J.-H. Xue, and G.-F. Yu, "Comprehensive analysis method of identifying and calibrating micro-seismic events attributes in coal and rock mass," Journal of Mining \& Safety Engineering, vol. 28, no. 1, pp. 61-65, 2011.

[10] Q.-J. Zhu, F.-X. Jiang, Y.-M. Yin, Z.-X. Yu, and J.-L. Wen, "Classification of mine microseismic events based on waveletfractal method and pattern recognition," Chinese Journal of Geotechnical Engineering, vol. 34, no. 11, pp. 2036-2042, 2012.

[11] F.-X. Jiang, Y.-M. Yin, Q.-J. Zhu, S.-X. Li, and Z.-X. Yu, "Feature extraction and classification of mining microseismic waveforms via multi-channels analysis," Journal of the China Coal Society, vol. 39, no. 2, pp. 229-237, 2014.
[12] P. Duplancic, Characterization of caving mechanism through analysis of stress and seismicity [Ph.D. thesis], University of Western Australia, Perth, Australia, 2001.

[13] Y. Abolfazlzadeh, Application of Seismic Monitoring in Caving Mines Case Study of Telfer Gold Mine, Laurentian University, Ontario, Canada, 2013.

[14] T. I. Urbancic, B. Feignier, and R. P. Young, "Influence of source region properties on scaling relations for $M<0$ events," Pure and Applied Geophysics, vol. 139, no. 3-4, pp. 721-739, 1992.

[15] M. R. Hudyma, D. Heal, and P. Mikula, "Seismic monitoring inmines-oldtechnology-newapplications," in Proceedings of the 1st Australasian Ground Control in Mining Conference, pp. 201-218, University of New South Wales, Sydney, Australia, 2003.

[16] J. Boatwright and J. B. Fletcher, "The partition of radiated energy between P and S waves," Bulletin of the Seismological Society of America, vol. 74, pp. 361-376, 1984.

[17] P. M. Shearer, Introduction to Seismology, Cambridge University Press, Cambridge, Mass, USA, 1999.

[18] L. J. Dong, X. B. Li, and G. Xie, "An analytical solution for acoustic emission source location for known $\mathrm{P}$ wave velocity system," Mathematical Problems in Engineering, vol. 2014, Article ID 290686, 8 pages, 2014.

[19] X. B. Li and L. J. Dong, "An efficient closed-form solution for acoustic emission source location in three-dimensional structures," AIP Advances, vol. 4, no. 2, Article ID 027110, 2014.

[20] L. J. Dong and X. B. Li, “Three-dimensional analytical solution of acoustic emission or microseismic source location under cube monitoring network," Transactions of Nonferrous Metals Society of China, vol. 22, no. 12, pp. 3087-3094, 2012.

[21] L. Dong and X. Li, "A microseismic/acoustic emission source location method using arrival times of PS waves for unknown velocity system," International Journal of Distributed Sensor Networks, vol. 2013, Article ID 307489, 8 pages, 2013.

[22] S. Weisberg, Applied Linear Regression, John Wiley \& Sons, Hoboken, NJ, USA, 3rd edition, 2005.

[23] L. Dong and X. Li, "Comprehensive models for evaluating rockmass stability based on statistical comparisons of multiple classifiers," Mathematical Problems in Engineering, vol. 2013, Article ID 395096, 9 pages, 2013.

[24] Å. Björck, Numerical Methods for Least Squares Problems, SIAM, Philadelphia, Pa, USA, 1996.

[25] A. R. Webb, Statistical Pattern Recognition, John Wiley \& Sons, 2nd edition, 2002.

[26] G. J. Mclachlan, Discriminant Analysis and Statistical Pattern Recognition, John Wiley \& Sons, Malvern, UK, 1992. 

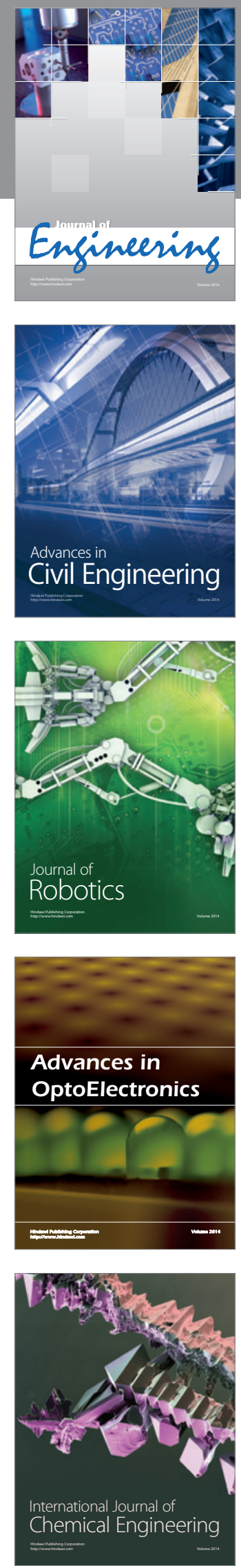

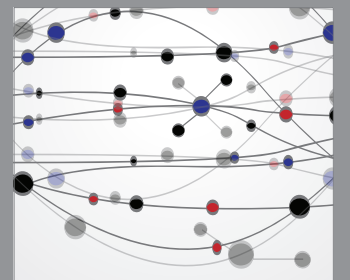

The Scientific World Journal
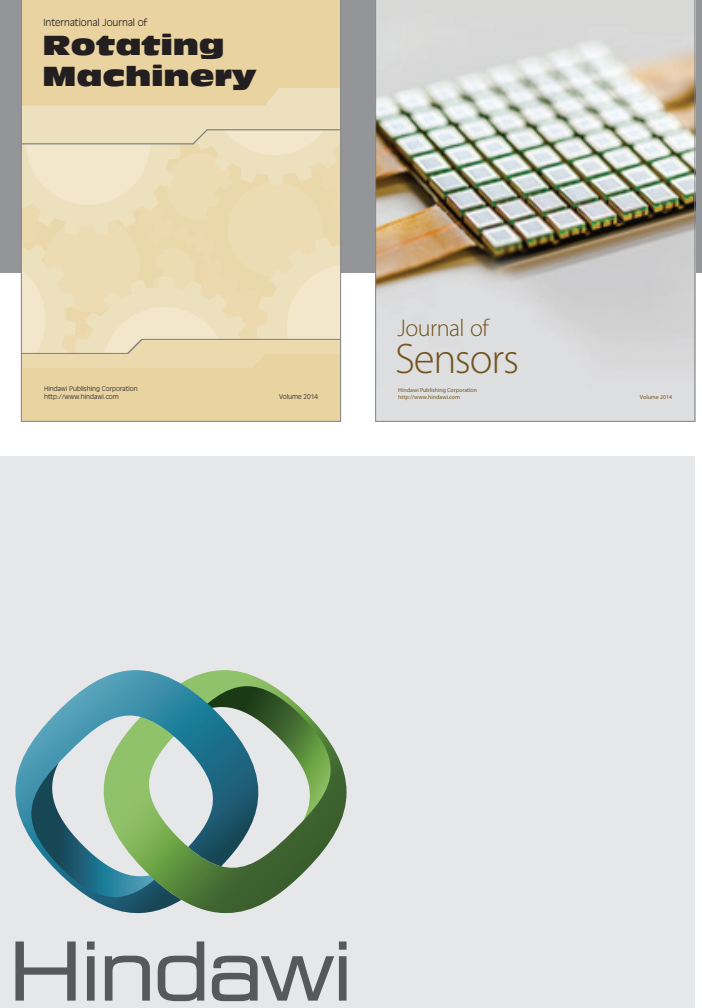

Submit your manuscripts at http://www.hindawi.com
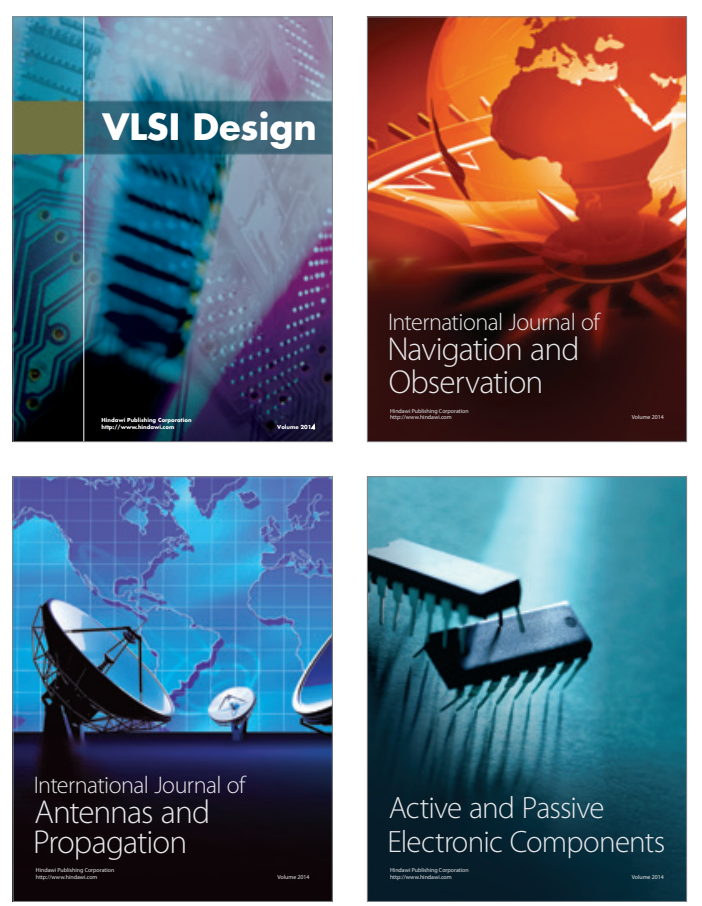
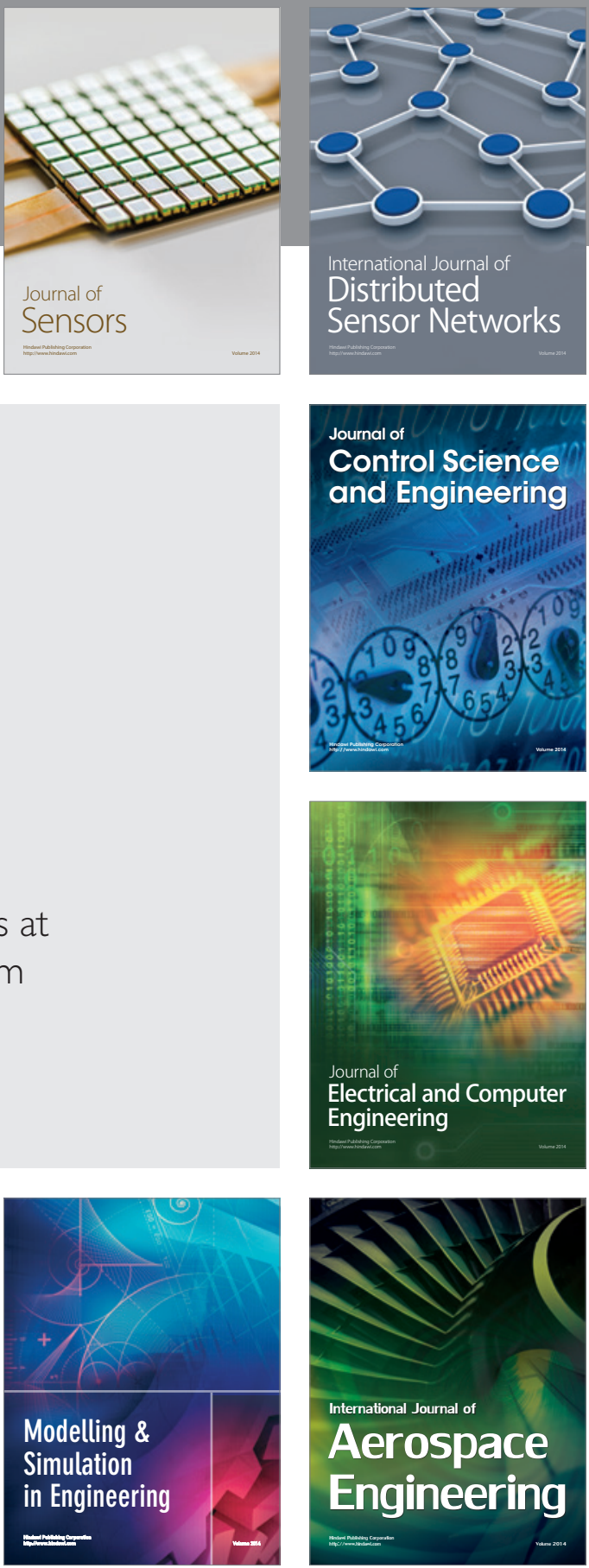

Journal of

Control Science

and Engineering
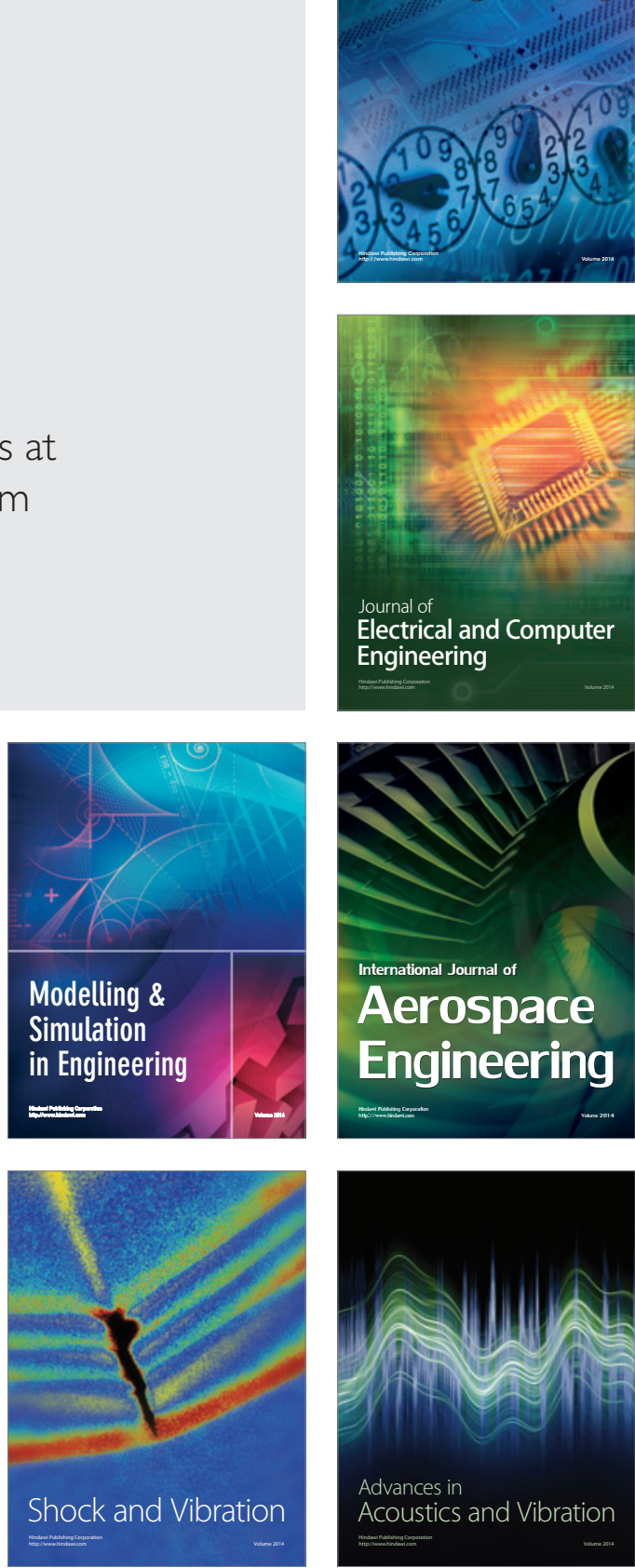